\title{
Evaluación nutricional e indicadores bioquimicos en jóvenes universitarios de la provincia de Sánchez Carrión, región La Libertad
}

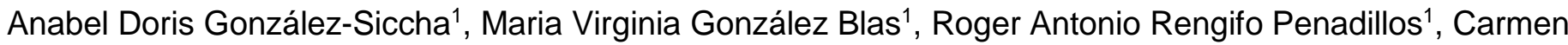 \\ Rosa Silva Correa1, Víctor Eduardo Villarreal La Torre ${ }^{1}$, George David González González² \\ ${ }^{1}$ Facultad de Farmacia y Bioquímica, Universidad Nacional de Trujillo, Av. Juan Pablo II s/n, Trujillo - Perú \\ ${ }^{2}$ Universidad Particular Antenor Orrego, Av. América Sur 3145 Monserrate, Trujillo -Perú
}

Recibido 15 de junio del 2018. Aceptado 21 de junio del 2018

DOI: https://doi.org/10.33017/RevECIPeru2018.0003/

\section{Resumen}

Objetivo: Determinar la evaluación nutricional e indicadores bioquímicos en jóvenes universitarios de la Provincia de Sánchez Carrión, Región La Libertad. Material y Método: Estudio descriptivo, de corte transversal y prospectivo. Participaron 29 jóvenes (26 mujeres y 03 varones), de 17-29 años de ambos sexos, habitantes de Huamachuco. Antes de su inclusión en el estudio, se requirió firma del consentimiento informado. Se determinaron las medidas antropométricas empleando variables: peso $(\mathrm{Kg})$, talla $(\mathrm{cm})$ e Índice de Masa Corporal. Se tomaron muestras de sangre por punción del pulpejo del dedo, se determinó hemoglobina (método Cianometahemoglobina) y hematocrito (microcentrifugación). Resultados: Los valores promedios fueron hemoglobina $=15.99 \pm 1.74$; hematocrito $=47,73 \pm 3,48$, IMC=23,66 $\pm 3,78$; edad $19.07 \pm .2,45$. Tuvieron IMC normal $72.42 \%$, sobrepeso $24.14 \%$ y obesidad $3.45 \%$ que podría deberse a una sobrenutrición y dieta rica en carbohidratos. El 62.07\% tuvo hemoglobina elevada con IMC normal (44.83\%), sobrepeso (13.79\%), obesidad $(3.45 \%)$, y el $31.03 \%$ con hemoglobina normal, sin significancia estadística $\left(x^{2}=0.684 ; P>0.05\right.$. El grupo de $17-$ 20 años de sexo femenino tuvo $62.07 \%$ de $\mathrm{Hb}$ elevada (policitemia fisiológica), pero la edad no influye en la concentración de $\mathrm{Hb} .\left(\mathrm{x}^{2}=5.151 ; \mathrm{P}>0.05\right)$ pero si el sexo $\left(\mathrm{x}^{2}=5.59 ; \mathrm{P}<0.05\right)$. El $68.96 \%$ presento hematocrito elevado con IMC normal (55.17\%), sobrepeso (13.79\%) y hematocrito normal (el $31.03 \%)$ sin significancia estadística $\left(x^{2}=3.192 ; P>0.05\right)$. Del $68.96 \%$ con hematocrito elevado, la mayoría según grupo etario fue de 1720 años $(62.07 \%),\left(x^{2}=0.787 ; P>0.05\right)$ y según el sexo fue $58.62 \%$ mujeres y el $10.34 \%$ varones $\left(x^{2}=1.487\right.$; $P>0.05)$. En las altas altitudes se estimula la eritropoyesis en compensación a la disminución parcial del oxígeno elevando la hemoglobina y hematocrito, denominado policitemia fisiológica. Se concluye que, el mayor porcentaje de jóvenes presentan valores elevados de hemoglobina y hematocrito con IMC normal debido a las altas altitudes que estimulan la eritropoyesis.

Descriptores: altas altitudes, hemoglobina, hematocrito, policitemia, IMC.

\begin{abstract}
Objective: To determine the nutritional evaluation and biochemical indicators in university students of the Province of Sánchez Carrión, La Libertad Region. Material and Method: Descriptive, cross-sectional study and prospective. Participants 29 young people (26 women and 03 men), 17-29 years of both sexes, inhabitants of Huamachuco. Prior to being included in the study, signed informed consent was required. It was determined the anthropometric measures using variables: weight $(\mathrm{Kg})$, height $(\mathrm{cm})$ and mass index bodily. Blood samples were taken by puncture of the finger pulp, hemoglobin was determined (method Cyanomethamoglobin) and hematocrit (microcentrifugation). Results: The average values were hemoglobin $=15.99 \pm 1.74$; hematocrit $=$ $47.73 \pm 3.48, \mathrm{BMI}=23.66 \pm 3.78$; age $19.07 \pm .2 .45$. They had normal BMl $72.42 \%$, overweight $24.14 \%$ and obesity $3.45 \%$ that could be due to overnutrition and diet rich in carbohydrates $62.07 \%$ had high hemoglobin with normal BMI (44.83\%), overweight (13.79\%), obesity (3.45\%), and 31.03\% with normal hemoglobin, without
\end{abstract}


statistical significance $(x 2=0.684, P>0.05$, the group of $17-20$ years of female sex had $62.07 \%$ high $\mathrm{Hb}$ (physiological polycythemia), but age does not influence the $\mathrm{Hb}$ concentration. $(\mathrm{x} 2=5.151 ; \mathrm{P}>0.05)$ but if the sex $(x 2=5.59 ; P<0.05) .68 .96 \%$ presented hematocrit elevated with normal BMI $(55.17 \%)$, overweight $(13.79 \%)$ and normal hematocrit $(31.03 \%)$ without significance statistics $(x 2=3.192 ; P>0.05)$. Of $68.96 \%$ with elevated hematocrit, the majority according to age group was $17-20$ years $(62.07 \%),(x 2=0.787, P>0.05)$ and according to sex was $58.62 \%$ women and $10.34 \%$ males $(x 2=1.487 ; \mathrm{P}>0.05)$. At high altitudes erythropoiesis is stimulated in compensation for the partial decrease of oxygen by elevating hemoglobin and hematocrit, called physiological polycythemia. It is concluded that, higher percentage of young people have high hemoglobin and hematocrit values with normal BMI due to at high altitudes that stimulate erythropoiesis.

Key words: high altitudes, hemoglobin, hematocrit, polycythemia, BMI, nutritional status.

\section{Introducción}

La evaluación nutricional es un indicador del estado de salud, permite determinar factores de riesgo en muchas enfermedades crónicas más prevalentes en la actualidad [1].

Los cambios de la economía alimentaria mundial se han reflejado en los hábitos alimentarios; por ejemplo, hay mayor consumo de alimentos muy energéticos con alto contenido de grasas, en particular grasas saturadas y en carbohidratos no refinados.

Estas características se combinan con la disminución del gasto energético que conlleva unos modos de vida ahorran trabajo en el hogar, disminución gradual de las tareas manuales físicamente exigentes en el trabajo, y dedicación preferente del tiempo de ocio a pasatiempos que no exigen esfuerzo físico [2].

En los países en desarrollo, las políticas alimentarias siguen concentrándose sólo en la desnutrición y no consideran la prevención de las enfermedades crónicas [3].

La evaluación del estado nutricional puede ser definida como la interpretación de la información obtenida a partir de estudios antropométricos, alimentarios, bioquímicos y clínicos [4].

El Índice de Masa Corporal (IMC), es un parámetro que indica el estado nutricional de la persona considerando el peso y la talla. [5]

La valoración bioquímica se realiza mediante indicadores hematológicos como la hemoglobina $(\mathrm{Hb})$, que es una proteína componente principal de los eritrocitos, representa el $32 \%$ de la masa total del glóbulo rojo y es el mejor índice para medir la capacidad de transporte de gases de la sangre.

La determinación de $\mathrm{Hb}$ mide la cantidad de la proteína que hay en un volumen de sangre y generalmente se expresa en $\mathrm{g} / \mathrm{L}[6]$.
Las cifras normales son variables y dependen de la edad, sexo, raza, altitud sobre el nivel del mar del lugar de residencia, ocupación, entre otras variables.

Los efectos de la exposición a la altura sobren diferentes variables hematológicas han sido de gran interés para investigadores en fisiología de la altura, el cual tiene su fundamento en los cambios en la presión atmosférica observados durante el ascenso a diferentes alturas sobre el nivel del mar.

A medida que se asciende a altitudes, la presión barométrica disminuye y por lo tanto la presión parcial de los gases también lo hace, siguiendo la ley de Dalton [7].

La hemoglobina en las personas que habitan en altas altitudes es mayor por el incremento en la producción de glóbulos rojos [8.]

El hematocrito (Hto), representa la proporción de glóbulos frente a la fracción plasmática en la sangre, es decir, es el volumen porcentual que ocupan los hematíes en la sangre, depende del número, forma y tamaño de los GR [9].

La disminución de la $\mathrm{Hb}$ y el Hto provocan anemia que puede deberse a deficiencia de hierro, malnutrición, enfermedad parasitaria [10].

Según la OMS: “...la salud de los adolescentes y jóvenes reviste una importancia decisiva para la sociedad, de ella depende el porvenir. Representan un factor considerable de progreso.

Es necesario poner su energía y su idealismo al servicio de todos. Las condiciones en las que viven y su modo de vida, los marcará para siempre...". [12].

\section{Problema:}

¿Cuál es su estado nutricional con las medidas antropométricas e indicadores bioquímicos de los jóvenes universitarios de La Provincia De Sánchez Carrión, Región La Libertad? 


\section{Objetivos}

1) Determinar las medidas estadísticas promedio de los valores de hemoglobina, hematocrito y sus medidas antropométricas: IMC, edad y sexo de los jóvenes universitarios de La Provincia de Sánchez Carrión, Región La Libertad.

2) Determinar los valores de hemoglobina según el IMC, grupo etario y sexo

3) Determinar los valores de hematocrito según el IMC, grupo etario y sexo.

4) Evaluar el estado nutricional mediante las medidas antropométricas según edad y sexo.

\section{Material y Método}

\subsection{Diseño y población de estudio}

Es una investigación descriptiva, transversal y prospectivo; en la cual se estudió un grupo de 29 jóvenes de 17-29 años de edad, de los cuales fueron 26 mujeres y 03 varones, habitantes de la Provincia de Sánchez Carrión, Región La Libertad.

\subsection{Aspectos éticos}

El criterio de inclusión fue que los jóvenes aceptarán participar en el estudio y firmarán el consentimiento informado. El criterio de exclusión fue que los jóvenes tengan alguna enfermedad durante la evaluación o que no quisieran participar en el estudio.Se realizó una encuesta de sus datos personales, edad, peso, talla y hábitos alimenticios [13].

\subsection{Indicadores antropométricos}

Se midió el peso utilizando una balanza cuya sensibilidad es de $100 \mathrm{~g}$, y talla de acuerdo a las recomendaciones aceptadas internacionalmente para la técnica antropométrica; previamente, los equipos fueron calibrados [4].

Con ambas medidas se calculó el índice de masa corporal (IMC) $(\mathrm{kg} / \mathrm{m} 2)$

Clasificación IMC: Bajo peso $(\mathrm{BP})<18,50$; $\operatorname{Normal}(N)=18,5-24,99 ; \quad$ Sobrepeso(S)>25-29,99; Obesidad $\geq 30,00$ [12].

\subsection{Evaluación bioquímica}

La muestra de sangre se obtuvo por punción del pulpejo del dedo, se determinó hemoglobina por el método de la Cianometahemoglobina y el hematocrito por microcentrifugación [13].

Los valores referencian de $\mathrm{Hb}$ en personas $>18$ años: Mujeres: 13-15.0 (g/dL), Hombres 14.0$17.0 \mathrm{~g} / \mathrm{dl}$; Hematocrito: mujeres $36-46 \%$ y en varones $40-50 \%$ [7] [14].

\subsection{Analisis estadistico}

Para el procesamiento y análisis de los datos se utilizó el programa SPSS versión 15.00 y Microsoft Office Excel para Windows, la estadística descriptiva (media aritmética, desviación estándar, valores mínimos, máximos y chi cuadrado).

\section{Resultados}

Tabla1: Medidas Estadísticas promedio de los valores de Hemoglobina, hematocrito con las medidas antropométricas IMC, edad y sexo en los jóvenes universitarios de la Provincia Sánchez Carrión, Región La Libertad

\begin{tabular}{lccccc}
\hline & \multicolumn{5}{c}{$\begin{array}{c}\text { JOVENES UNIVERSITARIOS DE LA } \\
\text { PROVINCIA SÁNCHEZ CARRIÓN }\end{array}$} \\
\cline { 2 - 6 } VARIABLE & $\mathrm{n} \quad \begin{array}{c}\text { VALOR } \\
\text { MÍNIMO }\end{array}$ & $\begin{array}{c}\text { VALOR } \\
\text { MÁXIMO }\end{array}$ & PROMEDIO & $\begin{array}{c}\text { DE } \\
\pm\end{array}$ \\
\hline $\begin{array}{l}\text { Hemoglobina } \\
\text { (g/dl) }\end{array}$ & 29.00 & 13.11 & 19.81 & 15.98 & 1.74 \\
\hline $\begin{array}{l}\text { Hematocrito } \\
\text { (\%) }\end{array}$ & 29.00 & 38.05 & 53.86 & 47.73 & 3.48 \\
\hline IMC (P/T2) & 29.00 & 18.73 & 38.67 & 23.66 & 3.77 \\
\hline EDAD (años) & 29.00 & 17.00 & 29.00 & 19.07 & 2.45 \\
\hline SEXO & 29.00 & $\mathrm{~F}$ & 26.00 & $\mathrm{M}$ & 3.00 \\
\hline
\end{tabular}

Tabla 2: Distribución de los valores de Hemoglobina con el IMC, edad y sexo en los jóvenes universitarios de la Provincia Sánchez Carrión, Región La Libertad

\begin{tabular}{|c|c|c|c|c|c|c|c|c|}
\hline \multirow{3}{*}{\multicolumn{2}{|c|}{$\begin{array}{l}\text { RELACIÓN DE LOS } \\
\text { CARACTERÍSTICOS }\end{array}$}} & \multicolumn{6}{|c|}{ Valores de Hemoglobina } & \multirow{3}{*}{$\begin{array}{c}\text { PRUEBA } \\
\text { ESTADISTICA } \\
\mathrm{X}^{2}\end{array}$} \\
\hline & & \multicolumn{2}{|c|}{ Normal } & \multicolumn{2}{|c|}{$\begin{array}{c}\text { Aumentado } \\
\mathrm{s}\end{array}$} & \multicolumn{2}{|c|}{ Total } & \\
\hline & & $n$ & $\%$ & $\mathrm{n}$ & $\%$ & $\mathrm{~N}$ & $\%$ & \\
\hline \multirow{4}{*}{$\begin{array}{c}2 a . \\
\text { IMC } \\
(\mathrm{Kg} / \mathrm{m} 2)\end{array}$} & Normal & 8.0 & 27.6 & 13.0 & 44.8 & 21.0 & 72.4 & $\mathrm{Hb}-\mathrm{IMC}$ \\
\hline & Sobrepeso & 3.0 & 10.3 & 4.0 & 13.8 & 7.0 & 24.1 & $X 2=0.684$ \\
\hline & Obesidad I & 0.0 & 0.0 & 1.0 & 3.4 & 1.0 & 3.4 & $P>0.05$ \\
\hline & TOTAL & 7.0 & 24.1 & 18.0 & 62.1 & 29.0 & 100.0 & \\
\hline \multirow{4}{*}{$\begin{array}{l}2 b . \\
\text { EDAD } \\
\text { (años) }\end{array}$} & $\begin{array}{l}17-20 \\
\text { años }\end{array}$ & 9.0 & 31.0 & 18.0 & 62.1 & 21.0 & 72.4 & $\mathrm{Hb}-\mathrm{EDAD}$ \\
\hline & $\begin{array}{l}21-24 \\
\text { años }\end{array}$ & 2.0 & 6.9 & 0.0 & 0.0 & 7.0 & 24.1 & $X 2=5.152$ \\
\hline & $\begin{array}{l}25-29 \\
\text { años }\end{array}$ & 1.0 & 3.4 & 0.0 & 0.0 & 1.0 & 3.4 & $P>0.05$ \\
\hline & TOTAL & 7.0 & 24.1 & 18.0 & 62.1 & 29.0 & 100.0 & \\
\hline \multirow{3}{*}{$\begin{array}{l}\text { 2.c } \\
\text { SEXO }\end{array}$} & Femenino & 8.0 & 27.6 & 18.0 & 62.1 & 26.0 & 89.7 & $\mathrm{Hb}$ - SEXO \\
\hline & Masculino & 3.0 & 10.3 & 0.0 & 0.0 & 3.0 & 10.3 & $X 2=5.590$ \\
\hline & TOTAL & 11.0 & 37.9 & 18.0 & 62.1 & 29.0 & 100.0 & $P<0.05$ \\
\hline
\end{tabular}

Tabla 3: Distribución de los valores de hematocrito con el IMC, edad y sexo en los jóvenes 
universitarios de la Provincia Sánchez Carrión, Región La Libertad

\begin{tabular}{|c|c|c|c|c|c|c|c|c|}
\hline \multirow{3}{*}{\multicolumn{2}{|c|}{$\begin{array}{l}\text { RELACIÓN DELOS } \\
\text { CARACTERISTICOS }\end{array}$}} & \multicolumn{6}{|c|}{ Valores de Hematocrito } & \multirow{3}{*}{$\begin{array}{c}\text { PRUEBA } \\
\text { ESTADISTICA } \\
\text { Chi Cuadrado } \\
\text { X2 }\end{array}$} \\
\hline & & \multicolumn{2}{|c|}{ Normal } & \multicolumn{2}{|c|}{ Aumentados } & \multicolumn{2}{|c|}{ Total } & \\
\hline & & $n$ & $\%$ & n & $\%$ & N & $\%$ & \\
\hline \multirow{4}{*}{$\begin{array}{l}\text { 3a. IMC } \\
\text { (Kg/m2) }\end{array}$} & Normal & 5.0 & 17.2 & 16.0 & 55.2 & 21.0 & 72.4 & $\mathrm{Hb} \cdot \mathrm{IMC}$ \\
\hline & Sobrepeso & 30 & 10.3 & 4.0 & 13.8 & 7.0 & 24.1 & $X 2=3.192$ \\
\hline & Obesidad I & 1.0 & 3.4 & 0.0 & 0.0 & 1.0 & 3.4 & $P>0.05$ \\
\hline & TOTAL & 9.0 & 31.0 & 20.0 & 69.0 & 29.0 & 100.0 & \\
\hline \multirow{4}{*}{$\begin{array}{l}\text { 3b. } \\
\text { EDAD } \\
\text { (años) }\end{array}$} & $17-20$ años & 8.0 & 27.6 & 18.0 & 62.1 & 26.0 & 89.7 & $\mathrm{Hb}-\mathrm{EDAD}$ \\
\hline & 21.24 años & 1.0 & 3.4 & 1.0 & 3.4 & 20 & 6.9 & $X 2=0.787$ \\
\hline & $25-29$ años & 0.0 & 0.0 & 1.0 & 3.4 & 1.0 & 3.4 & $P>0.05$ \\
\hline & TOTAL & 9.0 & 31.0 & 20.0 & 69.0 & 29.0 & 100.0 & \\
\hline \multirow{3}{*}{$\begin{array}{c}3 c . \\
\text { SEXO }\end{array}$} & Femenino & 9.0 & 31.0 & 17.0 & 58.6 & 26.0 & 89.7 & $\mathrm{Hb}-\mathrm{SEXO}$ \\
\hline & Masculino & 0.0 & 0.0 & 3.0 & 10.3 & 3.0 & 10.3 & $X 2=1.487$ \\
\hline & TOTAL & 9.0 & 31.0 & 20.0 & 69.0 & 29.0 & 100.0 & $P>0.05$ \\
\hline
\end{tabular}

Tabla 4: Distribución de los valores de hematocrito con el IMC, edad y sexo en los jóvenes universitarios de la Provincia Sánchez Carrión, Región La Libertad

\begin{tabular}{|c|c|c|c|c|c|c|c|c|c|c|c|c|c|c|c|}
\hline \multirow{4}{*}{\multicolumn{2}{|c|}{$\begin{array}{l}\text { RELACIÓN } \\
\text { DE } \\
\text { CARACTE } \\
\text { RÍSTICOS }\end{array}$}} & \multicolumn{13}{|c|}{ GRUPO ETARIO } & \multirow{4}{*}{$\begin{array}{r}\text { Total } \\
\%\end{array}$} \\
\hline & & \multicolumn{4}{|c|}{ 17-20 años } & \multicolumn{4}{|c|}{ 21-24 años } & & \multicolumn{4}{|c|}{25 - 29 años } & \\
\hline & & \multicolumn{2}{|c|}{$\mathrm{F}$} & \multicolumn{2}{|c|}{$M$} & \multicolumn{2}{|c|}{$\mathrm{F}$} & \multicolumn{2}{|c|}{$M$} & \multicolumn{2}{|r|}{$\mathrm{F}$} & \multicolumn{2}{|c|}{$M$} & & \\
\hline & & $\mathrm{n}$ & $\%$ & $\mathrm{n}$ & $\%$ & $\mathrm{n}$ & $\%$ & $\mathrm{n}$ & $\%$ & $\mathrm{n}$ & $\%$ & $\mathrm{n}$ & $\%$ & $\mathrm{~N}$ & \\
\hline \multirow{4}{*}{$\begin{array}{l}\text { IMC } \\
(\mathrm{Kg} / \\
\mathrm{m} 2)\end{array}$} & $\bar{N}$ & $\overline{18}$ & 62 & 1 & 3 & $\overline{0}$ & 0 & 1 & 3 & 0 & 0 & 1 & 3 & 21 & 72.4 \\
\hline & $S$ & 6 & 21 & 0 & 0 & 1 & 3 & 0 & 0 & 0 & 0 & 0 & 0 & 7 & 24.1 \\
\hline & 0 & 1 & 3 & 0 & 0 & 0 & 0 & 0 & 0 & 0 & 0 & 0 & 0 & 1 & 3.4 \\
\hline & TOT & 25 & 86 & 1 & 3 & 1 & 3 & 1 & 3 & 0 & 0 & 1 & 3 & 29 & 100. \\
\hline
\end{tabular}

\section{Interpretación de los resultados}

En la tabla 1, se observa que los valores promedio de $\mathrm{Hb} 15.98 \pm 1.74 \mathrm{~g} / \mathrm{dl}$, Hto $47.73 \pm 3.48 \%$, IMC $23.66 \pm 3.77 \mathrm{~kg} / \mathrm{m}^{2}$, se encuentran dentro del rango normal.

En la tabla 2, los valores de $\mathrm{Hb}$ relacionados con el IMC se presenta el $72.4 \%$ con IMC normal, de los cuales el $27.6 \%$ está con $\mathrm{Hb}$ normal y el $44.8 \% \mathrm{Hb}$ elevada.

En el caso de las personas con sobrepeso u obesidad, el tejido adiposo es el responsable de la inflamación crónica [15], la cual es expresada mediante la liberación de IL-6 y el TNF, ambos cofactores generan una mayor producción y liberación de hepcidina a partir del hígado, existiendo una correlación positiva entre la expresión de hepcidina-adipocito y el IMC [16].

La obesidad y sobrepeso son considerados como desórdenes inflamatorios crónicos y se postula que estarían asociados a anemia ferropénica debido a un aumento de la producción de la hormona hepcidina (Hpc) por el tejido adiposo [17]. Los valores de $\mathrm{Hb}$ no están asociados con el IMC
$(\mathrm{P}>0.05)$. El $62.10 \%$ presenta $\mathrm{Hb}$ elevada $(17-20$ años), del $41.4 \%$ de $\mathrm{Hb}$ normal, se distribuye el $31.0 \%$ (17-20 años), $6.9 \%$ (21-24 años) y el $3.4 \%$ (25-29 años) pero los valores de hemoglobina no están afectados por la edad $\left(x^{2}=5.152 ; P>0.05\right)$.

También se demuestra que la $\mathrm{Hb}$ elevada es mayor en mujeres (62.1\%) que, en varones, siendo estadísticamente significativo $\left(X^{2}=5.590 ; P<0.05\right)$.

En las altas altitudes, existe disminución de la presión parcial del oxígeno en el aire generando hipoxia hipobárica siendo los tejidos los más afectado, en compensación se estimula la eritropoyesis y el metabolismo del hierro (Fe), elevando los glóbulos rojos y la hemoglobina que intervienen en el transporte y utilización del oxígeno por los tejidos. [7].

En la tabla 3 , se muestra que el $55.2 \%$ tiene hematocrito elevado con su IMC normal y el $13.8 \%$ con sobrepeso $\left(x^{2}=3.192 ; P>0.05\right)$, siendo el $62.1 \%$ el grupo etario de 17-20 años, el más afectado con hematocrito elevado (y en menor proporción los grupos de 21-24 años y 25-29 años con 3.4\% respectivamente cada uno $\left(x^{2}=0.787 ; P>0.05\right)$. Del $69.0 \%$ de hematocrito elevado, corresponde el $58.6 \%$ al sexo femenino y $10.3 \%$ al masculino.

El Hto es la porción de volume total de la sangre ocupada por la masa de eritrocitos; representa, entonces, el porcentaje de la masa de eritrocitos en la sangre total y su cifra depende del tamaño del glóbulo rojo, por lo que no siempre refleja el número de hematíes, aunque sí es expresión de su concentración [18].

Se conoce que, a mayor altitud, el cuerpo humano posee adaptaciones a corto y largo plazo que le permiten compensar, en forma parcial, la falta de oxígeno, así la médula ósea sintetiza mayor cantidad de glóbulos rojos elevando los niveles de hematocrito. [19], [14].

En la tabla 4 se muestra la evaluación nutricional, el $86.2 \%$ son mujeres del grupo etario de $17-20$ años de las cuales el $62.1 \%$ presenta IMC normal, el $20.7 \%$ sobrepeso y el $3.45 \%$ obesidad. El sobrepeso y la obesidad puede deberse a que su dieta primaria es a base de carbohidratos con ello la sobrenutrición o falta de una dieta equilibrada que conduce a la obesidad y con ello problemas cardiovasculares y metabólicas [20].

\section{Conclusiones}

Los valores promedios de hemoglobina, hematocrito e índice de masa corporal se encuentran dentro de los valores normales. 
El mayor porcentaje presenta hemoglobina elevada $(62.1 \%)$, con un IMC normal siendo las mujeres de 17-20 años las más afectadas con policitemia fisiológica.

El mayor porcentaje presenta hematocrito elevado $(69.0 \%)$, con IMC normal siendo las mujeres de 1720 años las más afectadas con policitemia fisiológica.

En la evaluación nutricional, del $86.2 \%$ que son mujeres de $17-20$ años, el $62.1 \%$ presenta IMC normal, el $20.7 \%$ sobrepeso y el $3.45 \%$ obesidad.

\section{Conflictos de interés}

Los autores declaran no tener conflictos de interés en la publicación del presente artículo.

\section{Referencias}

[1] C. Martínez et al. Nutr Hosp. 20:3 (2005) 197-203.

[2] Organizacion Mundial de la Salud. Inf una Consult Mix Expert OMS/FAO. (2003) 1-86.

[ 3] R. Paredes, O. Orraca, E. Marimon, M. Casanova, L. Gonzalez. Rev Ciencias Medicas.18:2 (2014) 5.

[ 4] G. Figueroa. Fac Med Carrera Nutr. (2015) 1-105.

[ 5] P Ravasco, H. Anderson, F. Mardones. Nutr Hosp. 25:3 (2010) 57-66.

[ 6] A. Trompetero, E. Cristancho, W. Benavides et al. Memorias Rev Asoc Colomb Ciencias Biológicas. (2015) 27.
[7] A. Trompetero-Gonzalez et al. Univ Nac Colomb Fac Med. 63:4 (2014).

[8] G. Gonzales. Rev Peru Med Exp Salud Publica. 28:11 (2011) 92-100.

[9] J. Prieto. La Clínica y el laboratorio. (2006) 50-87

[10] Ministerio de salud. UNICEF. OPS. J Trop Pediatr. 13:4 (2014) 1-8.

[11] D. Hernández. Tesis Doctoral. Universidad de Granada, 2014.

[12] L. Hernández. Medigraphic Artemisa. (2001) 223-32.

[13] A. González Siccha et al. Manual de Prácticas de Laboratorio de Análisis clínicos. Edited por Univ Nac Trujillo. (2018) p.101.

[14] K. Saénz-Flor. Rev Fac Cien Med. 37 (2012) 55-64

[15] G. Blancas-Flores, J. Almanza-Pérez and R. López-Roa. Bol. Med. Hosp. Infant. Mex. 67 (2010) 03-12.

[16] S. Bekri et al. Gastroenterology. 131:3 (2006) 788-796.

[17] M. Sanad, M. Osman. Ital J Pediatr. 37:1 (2011).

[18] M. Forrellat-Barrios et al. Rev Cuba Hematol Inmunol y Hemoter. 26:4 (2010) 359-361.

[19] G. Gonzales and V. Tapia. Rev Med. 15:1 (2007) 80-93.

[20] M. Cruz et al. Reli y Soc. 57 ( 2013) 165-202.

\section{Autor correspondiente:}

Anabel Doris González-Siccha:

E-mail: agonzalez@unitru.edu.pe 